\title{
Computational evidence for hundreds of non-conserved plant microRNAs
}

\author{
Morten Lindow and Anders Krogh*
}

\author{
Address: Bioinformatics Centre, Institute of Molecular Biology, University of Copenhagen, Denmark \\ Email: Morten Lindow - morten@binf.ku.dk; Anders Krogh* - krogh@binf.ku.dk \\ * Corresponding author
}

Published: 13 September 2005

BMC Genomics 2005, 6:119 doi:10.1186/1471-2164-6-119

This article is available from: http://www.biomedcentral.com/I47I-2164/6/119

(C) 2005 Lindow and Krogh; licensee BioMed Central Ltd.

This is an Open Access article distributed under the terms of the Creative Commons Attribution License (http://creativecommons.org/licenses/by/2.0), which permits unrestricted use, distribution, and reproduction in any medium, provided the original work is properly cited.
Received: 14 April 2005

Accepted: 13 September 2005

\begin{abstract}
Background: MicroRNAs (miRNA) are small (20-25 nt) non-coding RNA molecules that regulate gene expression through interaction with mRNA in plants and metazoans. A few hundred miRNAs are known or predicted, and most of those are evolutionarily conserved. In general plant miRNA are different from their animal counterpart: most plant miRNAs show near perfect complementarity to their targets. Exploiting this complementarity we have developed a method for identification plant miRNAs that does not rely on phylogenetic conservation.
\end{abstract}

Results: Using the presumed targets for the known miRNA as positive controls, we list and filter all segments of the genome of length $\sim 20$ that are complementary to a target mRNA-transcript. From the positive control we recover 4 I (of 92 possible) of the already known miRNA-genes (representing 14 of 16 families) with only four false positives.

Applying the procedure to find possible new miRNAs targeting any annotated mRNA, we predict of 592 new miRNA genes, many of which are not conserved in other plant genomes. A subset of our predicted miRNAs is additionally supported by having more than one target that are not homologues.

Conclusion: These results indicate that it is possible to reliably predict miRNA-genes without using genome comparisons. Furthermore it suggests that the number of plant miRNAs have been underestimated and points to the existence of recently evolved miRNAs in Arabidopsis.

\section{Background}

MicroRNAs (miRNAs), 20-25 nucleotides in length, are involved in negative post transcriptional regulation in most multi-cellular organisms (for a review see e.g. $[1,2]$ ). The generality and importance of this recently discovered regulatory mechanism is gradually becoming apparent, and here we present computational evidence for new miRNAs indicating that their numbers are more abundant than previously believed, and argue that they play a major role in evolution.
Most of the miRNAs identified so far are conserved in other species, some remarkably well[3]. Previous computational screens for miRNA have relied on this evolutionary conservation to identify a few hundred putative miRNAs in vertebrates[4], C. elegans[5], and plants [6-8], and many have been experimentally confirmed (reviewed in [9]). However, these screens miss all miRNAs that have diverged since the last common ancestor of the genomes under comparison. A recent study using a combined bioinformatic and high-throughput experimental approach 
have identified 53 miRNAs not conserved beyond primates[10]. In plants, where comparisons have been between the distantly related $A$. thaliana (thale cress) and $O$. sativa (rice) genomes that diverged some 200 million years ago[11], it is probable that there are miRNAs which have escaped detection. Of the 112 Arabidopsis miRNAgenes currently registered[12], only 56 are conserved in the monocot rice (see methods section), indicating the existence of a substantial number of unconserved miRNAgenes. miRNA and short interfering RNAs (siRNA) are very similar in function, but different in biogenesis. According to the current nomenclature[13] both microRNAs (miRNAs) and short interfering RNAs (siRNAs) are 20-25 nucleotides long single stranded molecules that arise from processing of double stranded RNA (dsRNA) precursors. They are distinguished by the type of dsRNA they are excised from. While siRNAs come from long exogenous or endogenous dsRNA molecules (very long hairpins or RNA duplexes), mature miRNAs come from the stem region of shorter hairpins.

The mature miRNA or siRNA forms part of the RNA induced silencing complex (RISC) that binds to mRNAs. miRNA/siRNAs that bind with almost perfect complementarity to an mRNA often results in the cleavage of its target. Currently it seems that the higher the degree of complementarity to a target mRNA, the larger chance of that target being degraded. miRNAs with imperfect complementarity to a 3' untranslated region of a mRNA have been shown to inhibit translation of the mRNA[14,15]

When the base pairing between the miRNA and the target is incomplete it is non-trivial to identify targets for a miRNA [16-19]. In plants, however, most of the known miRNAs pair almost perfectly with one or more mRNAs, making it straightforward to identify likely plant targets (miRNAs often have more than one target). Using this observation it is possible to predict miRNA candidates in Arabidopsis that exhibit near perfect base pairing with the targets, without relying on homology to other organisms[20]. Here this idea is extended and refined to yield a highly specific screen that finds plant miRNAs in numbers much larger than previously thought.

\section{Results and discussion \\ Identification of non-conserved miRNAs}

The general approach is outlined in figure 1. Initially, a mRNA is compared with the genomic sequence to identify matching regions of 20-27 nucleotides with at most 2 mismatches (allowing 3 mismatches produced more than 10000 matches per mRNA). These are called micromatches, and the genomic part is referred to as a genomic match. An average mRNA gives rise to about 1000 such micromatches, the vast majority (often all) of which we assume are spurious non-miRNA hits. However, it is pos- sible, without comparing to other genomes, to filter the micromatches and achieve highly specific and fairly sensitive predictions of miRNA genes (Figure 1).

Six filters were used to identify a base set of genomic sequences as candidate miRNAs (with percentages of the initial micromatches that were remaining after each filter given in brackets): (1) they had high sequence complexity (26.9\%); (2) they had no overlap with annotated exons on the same or the opposite strand (3.3\%); (3) they had no overlap with repeat sequences defined by RepeatMasker $(2.6 \%)$; (4) the putative miRNA:mRNA duplex should be relatively stable $[17,21]$ with a calculated free energy of less than $-34 \mathrm{kcal} / \mathrm{mol}(0.20 \%)$; (5) they had no more than identical 10 copies in the genome $(0.19 \%)$, to eliminate repeated sequences not detected by standard repeatmasking; and (6) the miRNA was contained within a precursor structure that was similar to those observed in known Arabidopsis miRNA precursors, i.e. was predicted to be largely contained (at least 16 paired bases) within the stem of a double stranded stem-loop structure whose stem was predicted to have a free energy less than $-60 \mathrm{kcal} / \mathrm{mol}$, with at least 4 paired bases flanking the putative miRNA, and an intervening loop larger than 9 but less than 130 bases $(0.0002 \%)$.

Although the base set predictions have a low number of false positives (see below), they can be even more refined to identify a subset of the predictions with extra confidence, because the probability of more than one mRNA matching a falsely predicted miRNA is minimal, unless the matching mRNA-targets are close homologs (in which case the multiple targets do not add much extra confidence). Most of the known miRNA in Arabidopsis are thought to have multiple targets often within the same family of homologous proteins[22]. If a known miRNA only has targets in a highly conserved protein family this filter can however be expected to falsely eliminate them.

In order to check the validity of our approach we took the mRNA targets of the known miRNAs and set out to see if using these as queries we would be able to correctly identify the known miRNA-genes. Of the 112 precursor sequences registered in RFAM (ver 5.1), we were able to map 92 perfectly to the current RefSeq assembly (TIGR ver 5.0) of the Arabidopsis genome; the remaining precursors were excluded from the positive control set. Likely targets for Arabidopsis miRNAs have previously been predicted allowing for up to 3 mismatches[23]. Repeating this procedure we find that our known miRNAs match 142 different annotated mRNA*. These are the positive control targets (refered to a 'known targets') and many have been experimentally confirmed[24,25]. Initially, the 142 mRNAs in the positive control set yielded 359,976 micromatches after removal of low complexity sequences. 

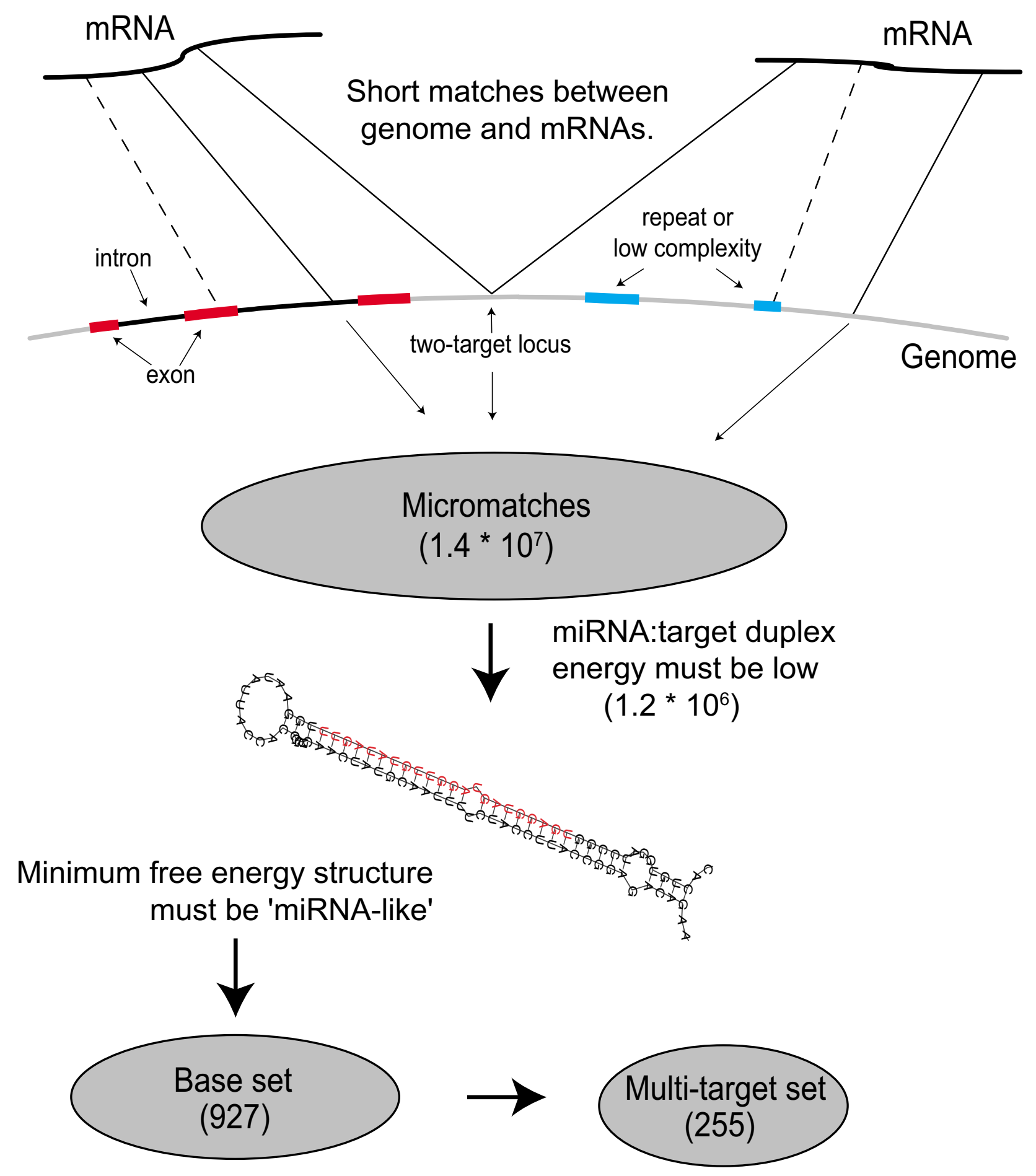

\section{Figure I}

Procedure for miRNA prediction. The number of matches between a mRNA and a segment of the genome (micromatches) after each step is shown in parenthesis. mRNAs are compared with the genomic sequence to identify matching regions of 20-27 nucleotides with at most 2 mismatches. Matches overlapping annotated exons, repeats or low-complexity regions are discarded. Additionally, the miRNA:mRNA-duplexes must be stable and the potential miRNAs must have a structure similar to known miRNAs to be included in the base set predictions. The multi-target set is a more reliable subset of those that have more than one target. See text for more details. 
However, the filtering procedure reduces this dramatically to 45 different loci (41 of which are already known) representing 16 different families ( 14 known). Assuming that the 'unknown' loci we find are false positives the procedure has $91 \%$ specificity and $45 \%$ sensitivity on the level of loci identified. Using the refinement step requiring more than one non-homologouos target only true positives are found, but at the expense of halving the sensitivity to $22 \%$. The validity of the estimates of specificity and sensitivity is discussed below.

\section{Hundreds of novel miRNAs}

Applying the micromatcher procedure to all 28860 mRNAs annotated in Arabidopsis identifies 592 miRNA candidate loci (480 families) in the base set (Additional file 1). In the final step this is reduced to a set of 90 (70 new) when more than one non-homologouos target per miRNA is required. This is called the multi-target set and is a subset of the base set.

All miRNA gene predictions, their targets (with some basic annotation) and the predicted secondary structure of the precursor are available as supplementary data [Additional file 1], and at our website[26]

Using public databases we were able to acquire evidence for the expression of a small number of the predictions, 9 in the base set overlap with RNA molecules recently sequenced in a large scale cloning effort of Arabidopsis small RNA ${ }^{4}, 109$ have significant matches to Arabidopsis ESTs and 52 of the predicted precursors contain a 20-mer sequence tag from the Arabidopsis MPSS database[27].

\section{Evolutionary conservation of the predicted miRNA-genes} From an evolutionary point of view, it would seem to be a lot easier to adapt 20 bases in a miRNA for a new target than to evolve a protein for a specific regulatory task.

For mammals it has been suggested that the more targets a microRNA has the more likely it is to be conserved[28] because of the additional constraints of having to match multiple targets.

Indeed also for plants: comparison of our predictions in Arabidopsis to two other plant species reveals that the more targets a miRNA is predicted to have, the more likely it is to be conserved (Figure 2). Although no Brassica species is yet completely sequenced and we had to use a conjunction of all single sequence Brassica entries from GenBank, significantly more of the predicted miRNAs are conserved in Brassica than in rice, indicating that many miRNAgenes have diverged beyond recognition since the divergence of monocots and dicots approximately 200 million years ago.

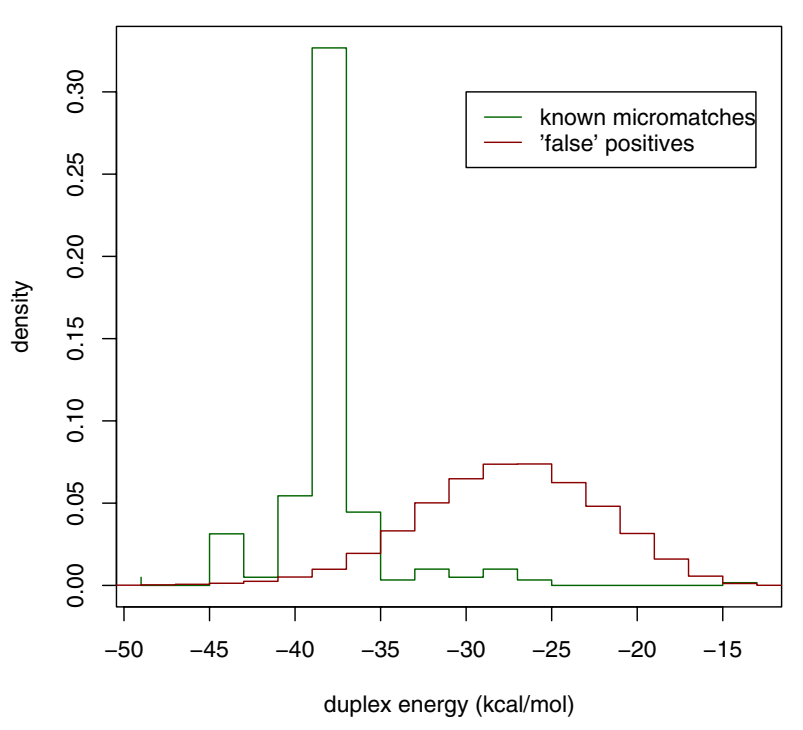

Figure 2

Duplex energy is a strong discriminant between true and false micromatches. The procedure was started with I 42 mRNAs targeted by known miRNAs. Micromatches were filtered for low-complexity, overlap with exons and repeats. Then the remaining micromatches were divided in two bins: true positives (green trace) that overlap with known miRNA genes and false positives (red trace) that do not.

Thus, we speculate that the highly conserved miRNAs are likely to be central regulators, often of many target mRNAs (imposing the evolutionary constraint to stay conserved), and are more likely to be highly expressed. Whereas more recently evolved miRNA would have fewer targets, and a more localized spatiotemporal expression, making them less likely to be detected by cloning efforts.

Since evolutionary conservation is part of many of the previous discovery procedures, it is likely that the set of known miRNAs is biased towards those that are conserved, and our data suggest that in fact, miRNAs evolve fast and are less conserved than e.g. protein-coding genes.

It has been proposed that some miRNAs originate from inverted duplication of target sequences, exemplified by the single locus miRNAs miR-161 and miR-163, which have precursors that show extended homology to the target mRNAs also outside the mature miRNA sequence[29]. However, our structural filters require that the match 
Table I: Summary of the results, starting with I36 mRNA targets to known miRNAs or all mRNAs, respectively. Numbers in parenthesis indicate the number of already known (RFAM) miRNA genes or families.

\begin{tabular}{|c|c|c|c|c|}
\hline & micromatches & miRNA genes found & distinct families & distinct targets \\
\hline \multicolumn{5}{|l|}{ Query: known targets } \\
\hline Baseset & 176 & $45(4 I)$ & $16(14)$ & 51 \\
\hline >I non-homologous target & 63 & $20(20)$ & $12(12)$ & 34 \\
\hline \multicolumn{5}{|l|}{ Query: all mRNAs } \\
\hline Baseset & 927 & 592 & 480 & 656 \\
\hline $\begin{array}{l}>\text { I target-homologous } \\
\text { target }\end{array}$ & 255 & 90 & 73 & 205 \\
\hline
\end{tabular}

between miRNA and target is in the range 20-25, effectively eliminating such miRNA with extended homology.

\section{Comparison to other studies}

Of the predicted 592 precursors in the base set, 29 overlap with the 92 predictions made by Bonnet et al.[30], and 4 of those by Wang et al.[8]. Thus, the different methods complement each other: The present method based on matching targets and miRNA is capable of finding nonconserved miRNAs, whereas the interspecies comparisons $[8,31]$ can find miRNAs without obvious targets.

The idea to use potential targets to find miRNA-genes has recently been employed in two other studies. Xie et al. [32] started by finding frequently occurring subsequences of human 3' UTR sequences conserved in other mammals and successfully searched the genome for new miRNA genes.

Moreover Adai and coworkers[33] published results in Arabidopsis using potential targets to find new miRNAgenes. However, our approach differs significantly from theirs in the way the matches (that we term micromatches) are analysed and the kind of conclusions that can be drawn: Adai et al. looks for a 'cluster' of miRNAgenes that target the same sequence of a mRNA, and then aligns the candidates in such a cluster, scoring the alignment high if it shows a characteristic pattern where the miRNA and miRNA* are more conserved than the intertwining sequence. Thus, their method is limited to finding miRNAs that occur more than once in the genome, presumably as a result of duplication events. Moreover as a postfilter, Adai et al. require conservation in rice to generate their short-list used for experimental validation. Also, Adai et al. do not make any estimation of the specificity of their computational procedure and are consequently unable to speculate about the number of miRNAs.

In contrast our method is independent of whether a candidate has been duplicated in the genome or is conserved across species. Instead our aggressive filtering on the structural properties of the precursor enables us to make highly specific prediction (judging from the results using targets for known miRNAs as queries).

The multi-target miRNAs have a total of 528 different mRNA targets, which are involved in a variety of functions, but there is a notable over-representation of proteins with transcription factor activity and receptor binding activity as well as involvement in developmental processes (false discovery rate $<0.001$, see Additional file $2)$. The predicted miRNA-genes are generally found scattered throughout the genome (Table 2). Unlike in mammals where 90 out of 232 miRNA-genes are within introns of protein coding genes [34], there is only one previously discovered Arabidopsis microRNA situated in an intron. This trend of plant microRNAs to be outside protein-coding genes also holds for our baseset predictions and even stronger for the multiple target predictions (Table 2).

Although estimating the sensitivity and specificity on the basis of the ability to correctly identify the small set of known miRNAs carries the danger of biasing, the presently most important concern must be not to massively overpredict new miRNA-genes. In constructing the filters we have therefore aim at high specificity at the expense of sensitivity. While false positives undoubtfully remain, the fact that the predictions share the properties of functional overrepresentation and bias of genomic location (properties not selected for in the filters) with known miRNAs provides independent indication that we indeed do not massively overpredict new miRNA-genes.

It is becoming evident that many regions between protein coding genes are transcribed (e.g. $[35,36])$. Indeed given the cases of miRNAs that have been suggested to regulate other miRNAs[37] or RNAs that guide methylation DNA[38], it would be interesting to extend our filtered intragenomic match approach to identify other possible miRNAs whose targets are not mRNAs. 
Table 2: The distribution of predicted miRNA-genes in relation to genomic features. IGR, intergenic region. The ratio of the number of bases annotated as intergenic vs. intron is 3.1 in the genome as a whole.

\begin{tabular}{lcc}
\hline Position of predicted miRNA genes & & \\
\hline & Base set & $>$ I target \\
\hline Total number of loci & 592 & 90 \\
In introns (sense strand) & 24 & 3 \\
in introns (antisense) & 18 & 2 \\
In intergenic regions (both strands) & 550 & 85 \\
$\quad$ Within 500 bases upstream of gene & 26 & 3 \\
$\quad$ Within 500 bases downstream of gene & 52 & 7 \\
Ratio IGR/introns & 14 & 18 \\
\hline
\end{tabular}

\section{Conclusion}

The present analysis predicts 71 new Arabidopsis miRNA genes with very few false positives (estimated specificity is $100 \%$ ) and over five hundred with an estimate of $9 \%$ false predictions. The procedure misses some real miRNAs, such as those encoded in untranslated regions of genes, those with very many targets (classified as repeats by our method), and those not fulfilling our strict structural constraints, and we believe that the real number could be several thousands. Although, the predictions should eventually be confirmed in the lab, our data suggest that the Arabidopsis genome encodes substantially more miRNA genes than previously thought, and that the number of miRNAs is comparable to the number of protein transcription factors. Our results also indicate that many miRNA are specific to small groups of related species and we speculate that they could play a part in speciation. Finally we find it unlikely that these conclusions are specific to plants, and we hypothesize that they extend to most other multicellular organisms.

\section{Methods}

\section{Sequences}

Arabidopsis genome and annotation were the RefSeq sequences based on the 5.0 version released by TIGR. Known miRNAs were from the 5.1 release of the microRNA registry[39].

\section{The micromatcher procedure}

\section{Finding all micromatches}

For each annotated spliced mRNA we exhaustively searched the genome for micromatches of length at least 20 with maximum 2 mismatches (no gaps allowed) using the suffixarray based program vmatch[40] (This search took 6 days on an Intel Xeon 2.2 Ghz machine running Linux).

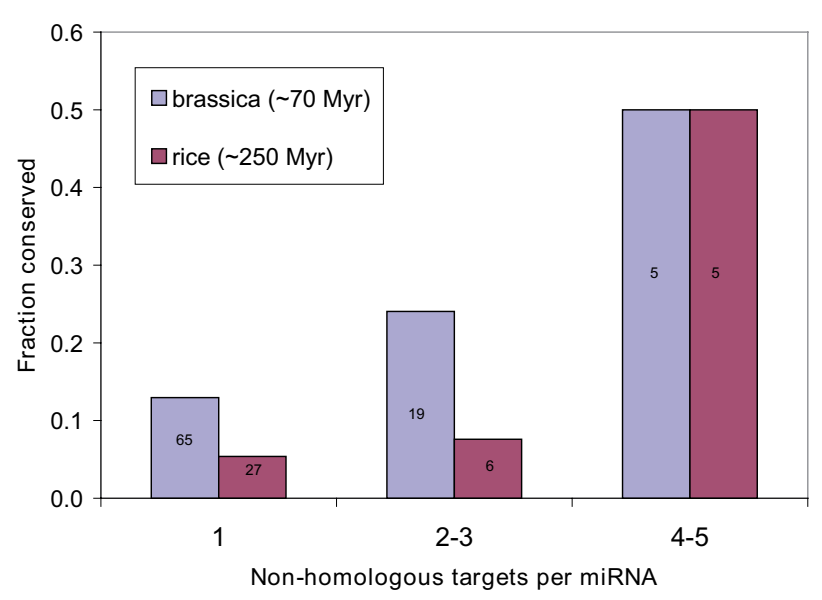

Figure 3

Multi target predictions tend to be better conserved. The precursor sequences of the predictions were used as queries for a blast search against rice (downloaded from tigr.org, March 2004) or brassica (downloaded from arabidopsis.org, August 2004), respectively. Columns show the proportion of miRNA predictions in Arabidopsis that were found to be conserved. Numbers refer to the actual number of conserved miRNA predictions.

Note about the positive control set of mRNAs: To select the positive control mRNA-targets we allow for 3 mismatches over the whole length of the mature miRNA; this potentially includes in the positive control set mRNAs that will be unable to recover the matching miRNA allowing only 2 mismatches over a length of 20 bases (the criterion used later). This discrepancy can lead to a too pessimistic estimation of the performance of procedure.

\section{Lowcomplexity filter}

Genomic micromatches not fulfilling a simple low complexity filter were discarded: 1) all four bases had to be present at least once, and 2) at most 11 of the three most frequent dinucleotides in the sequence were allowed.

\section{Duplex stability}

Using the program RNAcofold (Vienna RNA package[41]) the free energy change when a microRNA-candidate binds to a target site was calculated. Micromatches where this duplex energy is larger than $-34 \mathrm{kcal} / \mathrm{mol}$ were discarded.

\section{Long matches}

Micromatches longer than 26 residues were discarded. To ascertain that a micromatch was not part of a longer match, the two parts of the micromatch extended by 50 bases to each side were aligned with bl2seq (two sequence 
NCBI blast), and those with a match longer than 26 were discarded.

\section{Overlaps with known features and repeats}

A micromatch was discarded if it had any bases in common with annotated exons (including matches to the reverse strand of the exon) or repeats as determined by RepeatMasker[42] run with Arabidopsis specific repeat libraries (RepBase Update 8.12, RM database version 20040306).

\section{Copy number}

Additionally to traditional repeat-masking that relies on the identification of known repeats, we made an additional pragmatic repeat filter: We simply determined the number of times all candidate sequences occurs in the entire genome, and removed candidates with a copy number higher than 10 .

\section{Filtering on properties of the possible precursor}

In order to predict a possible precursor molecule, two genomic sequences around each micromatch were extracted: One starting 10 bases $5^{\prime}$ of the micromatch and extending 240 bases $3^{\prime}$ of the micromatch, and one with the extension lengths reversed. Each of these was treated independently in the following analysis. First the potential precursor sequence was folded with RNAfold[43] to find the minimum free energy structure These values are comparable, because all sequences are of almost equal length. Candidates with a folding free energy larger than $60 \mathrm{kcal} / \mathrm{mol}$ are discarded. This is a highly permissive filter. The mature miRNA has to be fully contained in a double stranded region of the precursor. The complementary part of the miRNA in this stem is denoted miRNA*. It is demanded that all base pairs between the miRNA and the miRNA* are pairing in the same direction opposite each other. The number of paired bases in the mature miRNA is required to be 16 or more.

In the known miRNA precursors, the stem is always longer than just the length of the mature miRNA. To find how far the stem of a candidate extends from the mature miRNA, we count how far inward towards the loop or outwards toward the ends of RNA-string the stem extends using the following algorithm: Moving out from the terminal basepair between miRNA and miRNA* a score of 1 is assigned for each base pair encountered and a score of -1 for each unpaired base. The extension is stopped when the current score is less than 5 lower than the maximum score so far. The last base pair is considered the terminus of the stem. Candidates with extensions less than 4 bases on either side of the mature miRNA were discarded. It was also required that the shortest number of bases between the miRNA and miRNA* were larger than 9 and less than 130.
Taken together these structural criteria constitute a highly selective, but somewhat conservative filter.

\section{Matches to ESTs and ASRP}

BLASTN was used to search all Arabidopsis ESTs downloaded from GenBank on September 27, 2004. Hits longer than 70 nucleotides with more than $95 \%$ identity between a predicted precursor and an EST were considered positive. Sequences cloned and sequenced as part of the Arabidopsis Small RNA Project (ASRP)[44], were downloaded from [45]. All matches at least 15 long with at most one mismatch with our predicted mature miRNAsequences were found using vmatch[46].

\section{Conservation in other genomes}

To determine how many of our predictions were conserved in other plant genomes, we blasted the predicted Arabidopsis precursors against the rice-genome and brassica sequence downloaded from [47]. A miRNA prediction was taken to be conserved if it had a significant ( $\mathrm{e}$-value < 0.01 ) blast hit containing the mature miRNA with no more than 2 mismatches and the homolog had flanking sequence capable of folding back on the mature miRNA with at least 15 base pairs between the miRNA and miRNA*.

\section{The number of non-homologous targets for a putative miRNA}

For all candidate microRNAs in the baseset matching more than one mRNA, we found the number of different non-homologous targets by performing single linkage clustering on the aminoacid sequences of the corresponding mRNAs using the program 'blastclust' from NCBI. Two proteins were considered homologous if they had more than $70 \%$ identity across at least $50 \%$ of the length.

\section{Clustering of micromatches into genomic loci}

Micromatches with genomic start position within 4 nucleotides were logically grouped into the same locus.

\section{Clustering of similar miRNA sequences into families}

We used the program vmatch[48] to align and perform single linkage clustering of the predicted mature miRNA sequences. Candidate pairs aligning over at least 17 bases, allowing an edit distance of 1 were grouped in the same family.

\section{Functional analysis of targets}

We obtained gene ontology annotation (GOSLIM) from [49]. From each GOSLIM category we constructed a $2 \times 2$ contingency table counting the number of targets vs nontargets with or without the GOSLIM annotation. We used $\mathrm{R}[50]$ to calculate p-values with Fisher's Exact Test and employed the package 'qvalue'[51] to correct for multiple testing setting a false discovery rate level at 0.001 . The 
results are included as [Additional file 2], along with the R-code used.

\section{Authors' contributions}

ML and AK designed the study. ML wrote the programs. $\mathrm{ML}$ and AK drafted the manuscript. Both authors read and approved the final manuscript.

\section{Additional material}

\section{Additional File 1}

Predicted miRNA genes. List of predicted miRNA-genes, their predicted targets, genomic location and graphics showing predicted structure of the precursors.

Click here for file

[http://www.biomedcentral.com/content/supplementary/14712164-6-119-S1.html]

\section{Additional File 2}

Functional analysis of the predicted miRNA targets. Analysis of overrepresented Gene Ontology terms among the mRNAs predicted to be targeted by miRNAs.

Click here for file

[http://www.biomedcentral.com/content/supplementary/14712164-6-119-S2.xls]

\section{Acknowledgements}

We wish to thank anonymous reviewers for helpful comments and suggestions.

\section{References}

I. He L, Hannon G]: MicroRNAs: small RNAs with a big role in gene regulation. Nat Rev Genet 2004, 5:522-53I.

2. Tomari Y, Zamore PD: Perspective: machines for RNAi. Genes Dev 2005, 19:517-529.

3. Floyd SK, Bowman JL: Gene regulation: ancient microRNA target sequences in plants. Nature 2004, 428:485-486.

4. Lim LP, Glasner ME, Yekta S, Burge CB, Bartel DP: Vertebrate microRNA genes. Science 2003, 299:1540.

5. Lim LP, Lau NC, Weinstein EG, Abdelhakim A, Yekta S, Rhoades MW, et al:: The microRNAs of Caenorhabditis elegans. Genes Dev 2003, 17:991-1008.

6. Jones-Rhoades MW, Bartel DP: Computational Identification of Plant MicroRNAs and Their Targets, Including a StressInduced miRNA. Mol Cell 2004, 1 4:787-799.

7. Bonnet E, Wuyts J, Rouze P, Van de PY: Detection of 9 I potential conserved plant microRNAs in Arabidopsis thaliana and Oryza sativa identifies important target genes. Proc Natl Acad Sci USA 2004, 101:1151I-11516.

8. Wang XJ, Reyes JL, Chua NH, Gaasterland T: Prediction and identification of Arabidopsis thaliana microRNAs and their mRNA targets. Genome Biol 2004, 5:R65.

9. Baulcombe D: RNA silencing in plants. Nature 2004, 43 I:356-363.

10. Bentwich I, Avniel A, Karov Y, Aharonov R, Gilad S, Barad O, et al. Identification of hundreds of conserved and nonconserved human microRNAs. Nat Genet 2005, 37:766-770.

1I. Nelson DR, Schuler MA, Paquette SM, Werck-Reichhart D, Bak S: Comparative genomics of rice and Arabidopsis. Analysis of 727 cytochrome $\mathbf{P 4 5 0}$ genes and pseudogenes from a monocot and a dicot. Plant Physiol 2004, 135:756-772.

12. Griffiths-Jones S: The microRNA Registry. Nucleic Acids Res 2004 , 32(Database):DI09-DIII.
13. Ambros V, Bartel B, Bartel DP, Burge CB, Carrington JC, Chen X, et al: A uniform system for microRNA annotation. RNA 2003, 9:277-279.

14. Doench JG, Petersen CP, Sharp PA: siRNAs can function as miRNAs. Genes Dev 2003, 17:438-442.

15. Doench JG, Sharp PA: Specificity of microRNA target selection in translational repression. Genes Dev 2004, I 8:504-5I I.

16. Doench JG, Sharp PA: Specificity of microRNA target selection in translational repression. Genes Dev 2004, I 8:504-5I I.

17. Enright AJ, John B, Gaul U, Tuschl T, Sander C, Marks DS: MicroRNA targets in Drosophila. Genome Biol 2003, 5:RI.

18. Stark A, Brennecke J, Russell RB, Cohen SM: Identification of Drosophila MicroRNA Targets. PLoS Biol 2003, I:E60.

19. Lewis BP, Shih IH, Jones-Rhoades MW, Bartel DP, Burge CB: Prediction of mammalian microRNA targets. Cell 2003, I I 5:787-798.

20. Adai A, Johnson C, Mlotshwa S, rcher-Evans S, Manocha V, Vance V, et al:: Computational prediction of miRNAs in Arabidopsis thaliana. Genome Res 2005, I5:78-9I.

21. Lewis BP, Shih IH, Jones-Rhoades MW, Bartel DP, Burge CB: Prediction of mammalian microRNA targets. Cell 2003, I I 5:787-798.

22. Rhoades MW, Reinhart BJ, Lim LP, Burge CB, Bartel B, Bartel DP: Prediction of plant microRNA targets. Cell 2002, I I 0:5। 3-520.

23. Rhoades MW, Reinhart BJ, Lim LP, Burge CB, Bartel B, Bartel DP: Prediction of plant microRNA targets. Cell 2002, I I 0:5 I 3-520.

24. Llave C, Xie Z, Kasschau KD, Carrington JC: Cleavage of Scarecrow-like mRNA targets directed by a class of Arabidopsis miRNA. Science 2002, 297:2053-2056.

25. Aukerman MJ, Sakai H: Regulation of flowering time and floral organ identity by a MicroRNA and its APETALA2-like target genes. Plant Cell 2003, I5:2730-274I.

26. [http://www.binf.ku.dk/users/morten/mimatcher/arabidopsis/mirn apredictions.html].

27. Meyers BC, Lee DK, Vu TH, Tej SS, Edberg SB, Matvienko M, et al: Arabidopsis MPSS. An online resource for quantitative expression analysis. Plant Physiol 2004, 135:80I-8I3.

28. Mattick JS, Makunin IV: Small regulatory RNAs in mammals. Hum Mol Genet 2005, I 4(Spec No I):RI2I-RI 32.

29. Allen E, Xie Z, Gustafson AM, Sung GH, Spatafora JW, Carrington JC: Evolution of microRNA genes by inverted duplication of target gene sequences in Arabidopsis thaliana. Nat Genet 2004, 36:1282-1290.

30. Bonnet E, Wuyts J, Rouze P, Van de PY: Detection of 9 I potential conserved plant microRNAs in Arabidopsis thaliana and Oryza sativa identifies important target genes. Proc Natl Acad Sci USA 2004, 101:1|151|-1|516.

3I. Bonnet E, Wuyts J, Rouze P, Van de PY: Detection of 9 I potential conserved plant microRNAs in Arabidopsis thaliana and Oryza sativa identifies important target genes. Proc Natl Acad Sci USA 2004, 101:II5II-II516.

32. Xie X, Lu J, Kulbokas EJ, Golub TR, Mootha V, Lindblad-Toh K, et al:: Systematic discovery of regulatory motifs in human promoters and 3' UTRs by comparison of several mammals. Nature 2005, 434:338-345.

33. Adai A, Johnson C, Mlotshwa S, rcher-Evans S, Manocha V, Vance V, et al.: Computational prediction of miRNAs in Arabidopsis thaliana. Genome Res 2005, I 5:78-9I.

34. Rodriguez A, Griffiths-Jones S, Ashurst JL, Bradley A: Identification of Mammalian microRNA Host Genes and Transcription Units. Genome Res 2004.

35. Cheng J, Kapranov P, Drenkow J, Dike S, Brubaker S, Patel S, et al: Transcriptional maps of 10 human chromosomes at 5-nucleotide resolution. Science 2005, 308: I | 49- I I54.

36. Stolc V, Samanta MP, Tongprasit W, Sethi H, Liang S, Nelson DC, et al.: Identification of transcribed sequences in Arabidopsis thaliana by using high-resolution genome tiling arrays. Proc Natl Acad Sci USA 2005, 102:4453-4458.

37. Lai EC, Wiel C, Rubin GM: Complementary miRNA pairs suggest a regulatory role for miRNA:miRNA duplexes. RNA 2004, 10:171-175.

38. Mathieu O, Bender J: RNA-directed DNA methylation. J Cell Sci 2004, II 7:4881-4888.

39. Griffiths-Jones S: The microRNA Registry. Nucleic Acids Res 2004 32(Database):D 109-DIII.

40. Kurtz S: The Vmatch large scale sequence analysis software. Ref Type: Computer Program . 4-12-2003 
41. Hofacker IL: Vienna RNA secondary structure server. Nucleic Acids Res 2003, 31 : 3429-343I.

42. [http://www.repeatmasker.org].

43. Hofacker IL: Vienna RNA secondary structure server. Nucleic Acids Res 2003, 31:3429-343I.

44. Xie Z, Johansen LK, Gustafson AM, Kasschau KD, Lellis AD, Zilberman $D$, et al.: Genetic and functional diversification of small RNA pathways in plants. PLOS Biol 2004, 2:EIO4.

45. [http://asrp.cgrb.oregonstate.edu/db]

46. Kurtz S: The Vmatch large scale sequence analysis software. Ref Type: Computer Program . 4-12-2003

47. [http://www.arabidopsis.org].

48. Kurtz S: The Vmatch large scale sequence analysis software. Ref Type: Computer Program . 4-12-2003

49. Berardini TZ, Mundodi S, Reiser L, Huala E, Garcia-Hernandez M, Zhang $P$, et al.: Functional annotation of the Arabidopsis genome using controlled vocabularies. Plant Physiol 2004, 135:745-755.

50. [http://www.r-project.org].

51. Storey JD: A direct approach to false discovery rates. Journal of the Royal Statistical Society, Series B 2002, 64:479-498.

Publish with Bio Med Central and every scientist can read your work free of charge

"BioMed Central will be the most significant development for disseminating the results of biomedical research in our lifetime. "

Sir Paul Nurse, Cancer Research UK

Your research papers will be:

- available free of charge to the entire biomedical community

- peer reviewed and published immediately upon acceptance

- cited in PubMed and archived on PubMed Central

- yours - you keep the copyright

Submit your manuscript here:

http://www.biomedcentral.com/info/publishing_adv.asp 